PROCEEDINGS OF THE

AMERICAN MATHEMATICAL SOCIETY

Volume 133, Number 2, Pages 511-518

S 0002-9939(04)07619-1

Article electronically published on August 4, 2004

\title{
A NOTE ON MEROMORPHIC OPERATORS
}

\author{
CHRISTOPH SCHMOEGER
}

(Communicated by Joseph A. Ball)

\begin{abstract}
Let $X$ be a complex Banach space and $T$ a bounded linear operator on $X$. $T$ is called meromorphic if the spectrum $\sigma(T)$ of $T$ is a countable set, with 0 the only possible point of accumulation, such that all the nonzero points of $\sigma(T)$ are poles of $(\lambda I-T)^{-1}$. By means of the analytical core $K(T)$ we give a spectral theory of meromorphic operators. Our results are a generalization of some results obtained by Gong and Wang (2003).
\end{abstract}

\section{INTRODUCTION AND TERMINOLOGY}

Throughout this paper, $X$ will denote an infinite-dimensional complex Banach space. By $\mathcal{L}(X)$ we denote the Banach algebra of all bounded linear operators on $X$. Let $T \in \mathcal{L}(X)$. The kernel and the range of $T$ will be denoted by $N(T)$ and $T(X)$, respectively. The spectrum, the set of eigenvalues, and the resolvent set of $T$ are denoted by $\sigma(T), \sigma_{p}(T)$ and $\rho(T)$, respectively. For the resolvent $(\lambda I-T)^{-1}$ we write $R_{\lambda}(T)(\lambda \in \rho(T))$.

The nullity $\alpha(T)$ of $T$ is the dimension of $N(T)$. The defect $\beta(T)$ of $T$ is the codimension of $T(X)$. The ascent $p(T)$ and the descent $q(T)$ are the extended integers given by

$$
\begin{array}{r}
p(T)=\inf \left\{n \geq 0: N\left(T^{n}\right)=N\left(T^{n+1}\right)\right\}, \\
q(T)=\inf \left\{n \geq 0: T^{n}(X)=T^{n+1}(X)\right\} .
\end{array}
$$

The infimum over the empty set is taken to be $\infty$. It follows from [4 Satz 72.3] that if $p(T)$ and $q(T)$ are both finite, then they are equal. If $\lambda_{0}$ is an isolated point in $\sigma(T)$, the spectral projection corresponding to $\lambda_{0}$ will denoted by $P_{\lambda_{0}}$. We have $X=P_{\lambda_{0}}(X) \oplus N\left(P_{\lambda_{0}}\right)$. From [4, Satz 101.2] we have the following characterization of the poles of $R_{\lambda}(T)$ :

Theorem 1. The complex number $\lambda_{0}$ is a pole of $R_{\lambda}(T)$ if and only if $0<$ $p\left(\lambda_{0} I-T\right)=q\left(\lambda_{0} I-T\right)<\infty$. In this case we have

$$
P_{\lambda_{0}}(X)=N\left(\left(\lambda_{o} I-T\right)^{p}\right) \text { and } N\left(P_{\lambda_{0}}\right)=\left(\lambda_{0} I-T\right)^{p}(X),
$$

where $p=p\left(\lambda_{0} I-T\right)$ is the order of the pole $\lambda_{0}$, and $\lambda_{0} \in \sigma_{p}(T)$.

Received by the editors August 15, 2003 and, in revised form, October 20, 2003.

2000 Mathematics Subject Classification. Primary 47A10, 47A11.

Key words and phrases. Meromorphic operators, analytical core.

(C)2004 American Mathematical Society 
We now list various classes of operators, which will be discussed in this note:

$$
\begin{aligned}
& \mathcal{F}(X)=\{T \in \mathcal{L}(X): \operatorname{dim} T(X)<\infty\} ; \\
& \mathcal{K}(X)=\{T \in \mathcal{L}(X): T \text { is compact }\} ; \\
& \Phi(X)=\{T \in \mathcal{L}(X): \alpha(T)<\infty, \beta(T)<\infty\} .
\end{aligned}
$$

Operators in $\Phi(X)$ are called Fredholm operators.

Let $T \in \mathcal{L}(X)$ and $\lambda \in \mathbb{C}$. $\lambda$ is called a Riesz point of $T$ if

$$
\alpha(\lambda I-T)=\beta(\lambda I-T)<\infty \text { and } p(\lambda I-T)=q(\lambda I-T)<\infty .
$$

$T \in \mathcal{L}(X)$ is called a Riesz operator if each $\lambda \neq 0$ is a Riesz point of $T$. We denote by $\mathcal{R}(X)$ the class of all Riesz operators in $\mathcal{L}(X)$.

We have the following characterization of Riesz operators (see [4, $\S 105]$ ):

Theorem 2. Let $T \in \mathcal{L}(X)$. Then:

$T \in \mathcal{R}(X) \Leftrightarrow$ each $\lambda_{0} \in \sigma(T) \backslash\{0\}$ is an isolated point of $\sigma(T)$ and $P_{\lambda_{0}} \in \mathcal{F}(X)$.

The class $\mathcal{M}(X)$ of meromorphic operators is defined as follows:

$$
\mathcal{M}(X)=\left\{T \in \mathcal{L}(X): \text { each } \lambda_{0} \in \sigma(T) \backslash\{0\} \text { is a pole of } R_{\lambda}(T)\right\} .
$$

We have the following inclusions:

$$
\mathcal{F}(X) \subseteq \mathcal{K}(X) \subseteq \mathcal{R}(X) \subseteq \mathcal{M}(X) .
$$

Two subclasses of $\mathcal{M}(X)$ are also considered in this note:

$$
\mathcal{Q}(X)=\{T \in \mathcal{L}(X): \sigma(T)=\{0\}\}
$$

and

$$
\mathcal{M}_{0}(X)=\{T \in \mathcal{M}(X): \sigma(T) \text { is finite }\} .
$$

An operator in $\mathcal{Q}(X)$ is called quasinilpotent.

In [5] Mbekhta introduced two important subspaces for $T \in \mathcal{L}(X)$ : the analytical core $K(T)$ of $T$ is defined by

$$
\begin{aligned}
K(T)=\{x \in X: & \text { there exist } c>0 \text { and a sequence }\left(x_{n}\right)_{n \geq 1} \text { in } X \text { such that } \\
& \left.T x_{1}=x, T x_{n+1}=x_{n} \text { and }\left\|x_{n}\right\| \leq c^{n}\|x\| \text { for all } n \in \mathbb{N}\right\} .
\end{aligned}
$$

Observe that if $Y$ is a closed subspace of $X$ such that $T(Y)=Y$, then $Y \subseteq K(T)$ ([8, Proposition 2]).

The subspace $H_{0}(T)$, defined by

$$
H_{0}(T)=\left\{x \in X: \lim _{n \rightarrow \infty}\left\|T^{n} x\right\|^{1 / n}=0\right\},
$$

is called the quasinilpotent part of $T$.

We close the section with the following definition: an operator $T \in \mathcal{L}(X)$ is said to have the single-valued extension property (SVEP) in $\lambda_{0} \in \mathbb{C}$ if for any holomorphic function $f: U \rightarrow X$, where $U$ is a neighbourhood of $\lambda_{0}$, with ( $\lambda I-$ $T) f(\lambda) \equiv 0$ for all $\lambda \in U$, the result is $f(\lambda) \equiv 0$. We say that $T$ has the SVEP if $T$ has the SVEP in each $\lambda \in \mathbb{C}$.

It is clear that each $T \in \mathcal{M}(X)$ has the SVEP. Furthermore, we have $\sigma(T) \backslash\{0\} \subseteq$ $\sigma_{p}(T)$ if $T \in \mathcal{M}(X)$ (see Theorem 1). 


\section{Preliminary Results}

In this section we collect some results which we need in the sequel.

Proposition 1. Let $T, S \in \mathcal{L}(X)$.

(1) $T(K(T))=K(T)$ and $T\left(H_{0}(T)\right) \subseteq H_{0}(T)$.

(2) $K(T) \subseteq T^{n}(X)$ and $N\left(T^{n}\right) \subseteq H_{0}(T)$ for all $n \in \mathbb{N}$.

(3) $N(\lambda I-T) \subseteq K(T)$ for all $\lambda \in \mathbb{C} \backslash\{0\}$.

(4) $H_{0}(T) \subseteq(\lambda I-T)(X)$ for all $\lambda \in \mathbb{C} \backslash\{0\}$.

(5) If $T S=S T$, then $H_{0}(T) \subseteq H_{0}(T S)$.

(6) $0 \in \rho(T) \Longleftrightarrow K(T)=X$ and $H_{0}(T)=\{0\}$.

Proof. (1) is shown in [6];

(2) is clear;

(3) if $x \in N(\lambda I-T)$, put $c=|\lambda|^{-1}$ and $x_{n}=|\lambda|^{-n} x(n \in \mathbb{N})$;

(4) is shown in 8 , Proposition 1];

(5) is clear;

(6) follows from (2) and(5).

Proposition 2. Let $T \in \mathcal{L}(X), \lambda_{0} \in \sigma(T)$ and $K\left(\lambda_{0} I-T\right)=\{0\}$. Then $\lambda_{0}$ is the only possible isolated point in $\sigma(T)$.

Proof. Corollary 1.3 in [7].

Proposition 3. Suppose that $T \in \mathcal{L}(X)$ has the SVEP in $\lambda_{0}=0$.

(1) If $q(T)<\infty$, then $p(T)=q(T)$.

(2) 0 is a pole of $R_{\lambda}(T)$ if and only if $0<q(T)<\infty$.

Proof. (1) is shown in [8] Proposition 3].

(2) If 0 is a pole of $R_{\lambda}(T)$, then $0<q(T)<\infty$ by Theorem 2. If $0<q(T)<\infty$, it follows from (1) that $0<p(T)=q(T)<\infty$ : Theorem 2 shows now that 0 is a pole of $R_{\lambda}(T)$.

Proposition 4. Let $T \in \mathcal{L}(X) .0$ is an isolated point of $\sigma(T)$ if and only if $K(T)$ is closed, $X=K(T)+H_{0}(T)$ and $K(T) \cap H_{0}(T)=\{0\}$. In this case,

$$
P_{0}(X)=H_{0}(T) \text { and } N\left(P_{0}\right)=K(T) .
$$

Proof. Proposition 4 and Theorem 4 in 8 .

Notation. If $T \in \mathcal{L}(X)$ and if $Y$ is a $T$-invariant subspace of $X$, then $\left.T\right|_{Y}$ means the restriction of $T$ to $Y$.

Proposition 5. Let $T \in \mathcal{L}(X)$ and $\lambda_{0} \in \mathbb{C} \backslash\{0\}$. If $\lambda_{0}$ is an isolated point of $\sigma(T)$, then

$H_{0}\left(\lambda_{0} I-T\right)$ is a closed T-invariant subspace and $\sigma\left(\left.T\right|_{H_{0}\left(\lambda_{0} I-T\right)}\right)=\left\{\lambda_{0}\right\}$.

Proof. By Proposition 1(1) and Proposition 4, T( $\left.H_{0}\left(\lambda_{0} I-T\right)\right) \subseteq H_{0}\left(\lambda_{0} I-T\right)$ and $H_{0}\left(\lambda_{0} I-T\right)=P_{\lambda_{0}}(X)$, thus $H_{0}\left(\lambda_{0} I-T\right)$ is closed and $T$-invariant. From [4] Satz 100.1] we get $\sigma\left(\left.T\right|_{H_{0}\left(\lambda_{0} I-T\right)}\right)=\left\{\lambda_{0}\right\}$.

The next result generalizes Proposition 2.4 in [7]. 
Proposition 6. Suppose that $T \in \mathcal{L}(X)$ has the $S V E P, \lambda_{0} \in \mathbb{C} \backslash\{0\}, \lambda_{0} \in \rho(T)$ or $\lambda_{0}$ is an isolated point of $\sigma(T)$ and that

$$
H_{0}\left(\lambda_{0} I-T\right)+H_{0}(T)=X .
$$

Then $0 \in \rho(T)$ or 0 is an isolated point of $\sigma(T)$.

Proof. Put $Y=H_{0}\left(\lambda_{0} I-T\right)$ ). If $\lambda_{0} \in \rho(T)$, then $Y=\{0\}$ (by Proposition 1(6)); thus,

$$
(\lambda I-T)(Y)=Y \text { for all } \lambda \in \mathbb{C} .
$$

If $\lambda_{0} \in \sigma(T)$, then, by Proposition 5, there exists $\rho>0$ such that

$$
(\lambda I-T)(Y)=Y \text { for }|\lambda|<\rho .
$$

Therefore we have in both cases that there is some $\rho>0$ with $(\lambda I-T)(Y)=Y$ for $|\lambda|<\rho$.

Now take $\lambda \in \mathbb{C}$ with $0<|\lambda|<\rho$. Then

$$
H_{0}(\lambda I-T)=Y \subseteq(\lambda I-T)(X),
$$

thus $X=H_{0}\left(\lambda_{0} I-T\right)+H_{0}(T) \subseteq(\lambda I-T)(X)+H_{0}(T)$.

Since $H_{0}(T) \subseteq(\lambda I-T)(X)$ (by Proposition 1(4)),

$$
X=(\lambda I-T)(X),
$$

therefore $q(\lambda I-T)=0$ for $0<|\lambda|<\rho$. Since $T$ has the SVEP, we get from Proposition 3(1) that $p(\lambda I-T)=q(\lambda I-T)=0$ for $0<|\lambda|<\rho$. Hence $\{\lambda \in$ $\mathbb{C}: 0<|\lambda|<\rho\} \subseteq \rho(T)$.

Corollary 1. Suppose that $T \in \mathcal{L}(X)$ has the SVEP, $\lambda_{0} \in \mathbb{C} \backslash\{0\}, \lambda_{0} \in \rho(T)$ or $\lambda_{0}$ is an isolated point of $\sigma(T)$ and that

$$
H_{0}(T)=K\left(\lambda_{0} I-T\right) .
$$

Then $0 \in \rho(T)$ or 0 is an isolated point of $\sigma(T)$.

Proof. If $\lambda_{0} \in \rho(T)$, then $K\left(\lambda_{0} I-T\right)=X$ and $H_{0}\left(\lambda_{0} I-T\right)=\{0\}$ (Proposition $1(6))$. Thus

$$
X=K\left(\lambda_{0} I-T\right)+H\left(\lambda_{0} I-T\right)
$$

hence

$$
X=H_{0}(T)+H_{0}\left(\lambda_{0} I-T\right) .
$$

If $\lambda_{0} \in \sigma(T)$, then, by Proposition 4,

$$
X=K\left(\lambda_{0} I-T\right)+H\left(\lambda_{0} I-T\right),
$$

therefore

$$
X=H_{0}(T)+H_{0}\left(\lambda_{0} I-T\right) .
$$

Thus we have in both cases that $X=H_{0}(T)+H_{0}\left(\lambda_{0} I-T\right)$. Now use Proposition 6 .

Remark. Corollary 1 generalizes [7, Corollary 2.5]. 


\section{Meromorphic operators}

In this section we present the main results of this paper. The first result deals with Riesz operators and generalizes Theorem 2.6 in [7].

Theorem 3. Let $T \in \mathcal{R}(X)$. The following assertions are equivalent:

(1) 0 is a pole of $R_{\lambda}(T)$;

(2) there exists $q \in \mathbb{N}$ such that $T^{q} \in \mathcal{F}(X)$;

(3) there exists $n \in \mathbb{N}$ with $K(T)=T^{n}(X)$;

(4) $q(T)<\infty$.

Proof. (1) $\Leftrightarrow(2)$ : 4, Aufgabe 105.2].

$(2) \Rightarrow(3)$ : Since $T^{q+k}(X) \subseteq T^{q}(X)$ for $k \geq 0$ and $\operatorname{dim} T^{q}(X)<\infty$, we get $q \leq q(T)<\infty$. Put $n=q(T)$. Then $\operatorname{dim} T^{n}(X)<\infty$, hence $T^{n}(X)$ is closed. Furthermore $T\left(T^{n}(X)\right)=T^{n+1}(X)=T^{n}(X)$. Proposition 2 in [8] implies now that $T^{n}(X) \subseteq K(T)$. Therefore $K(T)=T^{n}(X)$, by Proposition $1(2)$.

$(3) \Rightarrow(4)$ : From $T^{n+1}(X)=T\left(T^{n}(X)\right)=T(K(T))$ and $T(K(T))=K(T)$ (Proposition 1(1)) we derive $T^{n+1}(X)=T^{n}(X)$, thus $q(T) \leq n<\infty$.

$(4) \Rightarrow(1)$ : Since $T$ has the SVEP, it follows from Proposition 3(2) that 0 is a pole of $R_{\lambda}(T)$.

Remark. The above proof shows that if $T \in \mathcal{L}(X)$ has the SVEP in $\lambda_{0}=0$ and if $0 \in \sigma(T)$, then the assertions (1), (3) and (4) in Theorem 3 are equivalent (for the implication (1) $\Rightarrow$ (3) use Theorem 1 and Proposition 4).

Our next result generalizes Theorem 2.1 in [7].

Theorem 4. Let $T \in \mathcal{M}(X)$. Then:

$0 \in \rho(T)$ or 0 is an isolated point of $\sigma(T) \Leftrightarrow K(T)$ is closed.

Proof. " $\Rightarrow$ ": Proposition 1(6) and Proposition 4 show that $K(T)$ is closed if $0 \in$ $\rho(T)$ or 0 is an isolated point of $\sigma(T)$.

"६": Case 1: $K(T)=\{0\}$. Proposition $1(6)$ shows that $0 \in \sigma(T)$. Proposition 2 implies then that 0 is the only possible isolated point of $\sigma(T)$. Since $T \in \mathcal{M}(X)$ we get $\sigma(T)=\{0\}$ (hence $T \in \mathcal{Q}(X)$ ).

Case 2: $K(T) \neq\{0\}$. Since $K(T)$ is closed, $K(T)$ is a Banach space. Put $T_{0}:=\left.T\right|_{K(T)}$ and $I_{0}=\left.I\right|_{K(T)}$. Use Proposition 1(1) to get

$$
T_{0} \in \mathcal{L}(K(T)) \text { and } q\left(T_{0}\right)=0 .
$$

Since $T$ has the SVEP, $T_{0}$ has the SVEP.

From Proposition 3(1) we therefore derive $p\left(T_{0}\right)=q\left(T_{0}\right)=0$, hence $0 \in \rho\left(T_{0}\right)$. Thus there is $\rho>0$ such that $\{\lambda \in \mathbb{C}:|\lambda|<\rho\} \subseteq \rho\left(T_{0}\right)$. Now take $\lambda \in \mathcal{C}$ with $0<|\lambda|<\rho$. Then $N(\lambda I-T) \subseteq K(T)$ (Proposition 1(3)), thus

$$
N(\lambda I-T)=N\left(\lambda I_{0}-T_{0}\right)=\{0\},
$$

hence $\lambda \notin \sigma_{p}(T)$. Since $\lambda \neq 0$ and $T \in \mathcal{M}(X), \lambda \notin \sigma(T)$. Therefore $\{\lambda \in \mathbb{C}: 0<$ $|\lambda|<\rho\} \subseteq \rho(T)$.

We proceed with a corollary that generalizes Corollary 2.2 in [7].

Corollary 2. Let $T \in \mathcal{M}(X)$. Then

(1) $K(T)=\{0\} \Leftrightarrow T \in Q(X)$;

(2) $K(T)$ is closed and $K(T) \neq\{0\} \Leftrightarrow T \in \mathcal{M}_{0}(X) \backslash \mathcal{Q}(X)$;

(3) $K(T)$ is not closed $\Leftrightarrow T \notin \mathcal{M}_{0}(X)$. 
Proof. (1) We have seen in the proof of Theorem 4 that $K(T)=\{0\}$ implies $T \in \mathcal{Q}(X)$.

Now let $T \in \mathcal{Q}(X)$. Remarque 1.1 in [6] shows that $H_{0}(T)=X$. Since $H_{0}(T) \cap$ $K(T)=\{0\}$, by Proposition 4, we derive $K(T)=\{0\}$.

(2) " $\Rightarrow$ ": (1) gives $T \notin \mathcal{Q}(X)$. Theorem 4 shows that $0 \in \rho(T)$ or 0 is an isolated point of $\sigma(T)$. Therefore, since $T \in \mathcal{M}(X), \sigma(T)$ is finite, hence $T \in \mathcal{M}_{0}(X)$.

" $\Leftarrow$ ": From (1) we get $K(T) \neq\{0\}$. Since $\sigma(T)$ is finite, we see that $0 \in \rho(T)$ or 0 is an isolated point of $\sigma(T)$. Theorem 4 implies then that $K(T)$ is closed.

$(3)$ " $\Rightarrow$ ": By Theorem $4,0 \in \sigma(T)$ and 0 is not an isolated point of $\sigma(T)$, thus $T \notin \mathcal{M}_{0}(X)$.

" $\Leftarrow$ ": Since $T \in \mathcal{M}(X) \backslash \mathcal{M}_{0}(X), 0$ is a point of accumulation of $\sigma(T)$, thus $K(T)$ is not closed by Theorem 4 .

We denote by $X^{*}$ the dual space of $X$ and by $T^{*}$ the adjoint of $T \in \mathcal{L}(X)$.

Proposition 7. Let $T \in \mathcal{L}(X)$, and suppose that $T$ and $T^{*}$ have the $S V E P$ in 0 . Then:

$$
T \in \Phi(X) \Leftrightarrow 0 \text { is a Riesz point of } T \text {. }
$$

Proof. The implication " $\Leftarrow$ " follows from the definition of a Riesz point.

Now suppose that $T \in \Phi(X)$. Since $T$ has the SVEP in 0 , it follows from [3. Theorem 15] that $p(T)<\infty$. Satz 82.1 in [4 gives $T^{*} \in \Phi\left(X^{*}\right)$. Since $T^{*}$ has the SVEP in 0 , we have $q(T)<\infty$ by [3, Corollary 16]. Hence $p(T)=q(T)<\infty$. Satz 72.5 in [4] implies now that $\alpha(T)=\beta(T)$.

Corollary 3. For $T \in \mathcal{M}(X)$ the following assertions are equivalent:

(1) $K(T)$ is closed and $\operatorname{codim} K(T)<\infty$;

(2) $K(T)$ is closed and $\operatorname{dim} H_{0}(T)<\infty$;

(3) 0 is a Riesz point of $T$;

(4) $T \in \Phi(X)$.

Proof. Since $T \in \mathcal{M}(X)$ and $\sigma\left(T^{*}\right)=\sigma(T), T$ and $T^{*}$ have the SVEP. Proposition 7 shows then that (3) and (4) are equivalent.

Now suppose that $K(T)$ is closed. By Theorem $4,0 \in \rho(T)$ or 0 is an isolated point of $\sigma(T)$. Now use Proposition 1(6) and Proposition 4 to derive

$$
X=K(T)+H_{0}(T) \text { and } K(T) \cap H_{0}(T)=\{0\} .
$$

Hence $\operatorname{dim} H_{0}(T)=\operatorname{codim} K(T)$. Therefore (1) and (2) are equivalent.

Now we show that (2) implies (3): By Theorem $4,0 \in \rho(T)$ or 0 is an isolated point of $\sigma(T)$. If $0 \in \rho(T)$, then 0 is a Riesz point of $T$. Hence suppose that $0 \in \sigma(T)$. By Proposition 4, $P_{0}(X)=H_{0}(T)$, thus $P_{0} \in \mathcal{F}(X)$. [4, Satz 105.2] shows now that 0 is a Riesz point of $T$.

It remains to show that (3) implies (2):

Case 1: $0 \in \rho(T)$. By Proposition 1(6), $K(T)=X$ and $H_{0}(T)=\{0\}$. Hence $K(T)$ is closed and $\operatorname{dim} H_{0}(T)<\infty$.

Case 2: $0 \in \sigma(T)$. Since 0 is a Riesz point of $T, 0$ is an isolated point of $\sigma(T)$ and $P_{0} \in \mathcal{F}(X)$, by Satz 105.2 in [4]. From Proposition 4 and Theorem 4 it follows that $\operatorname{dim} H_{0}(T)=\operatorname{dim} P_{0}(X)<\infty$ and that $K(T)$ is closed.

Corollary 4. For $T \in \mathcal{M}(X)$ the following assertions are equivalent:

(1) $\operatorname{dim} K(T)<\infty$;

(2) $T \in \mathcal{R}(X) \cap \mathcal{M}_{0}(X)$. 
Proof. (1) $\Rightarrow(2)$ : Since $\operatorname{dim} X=\infty$ and $\operatorname{dim} K(T)<\infty$, it follows from Proposition $1(6)$ that $0 \in \sigma(T)$. Corollary 2 shows that $T \in \mathcal{M}_{0}(X)$.

Now take $\lambda \in \mathbb{C} \backslash\{0\}$. Since $T \in \mathcal{M}(X), \lambda \in \rho(T)$ or $\lambda$ is a pole of $R_{\lambda}(T)$, thus $p(\lambda I-T)=q(\lambda I-T)<\infty$. By Proposition 1(3), $N(\lambda I-T) \subseteq K(T)$, thus $\alpha(\lambda I-T)<\infty$. Satz 72.5 in [4] implies now that

$$
\beta(\lambda I-T)=\alpha(\lambda I-T)<\infty .
$$

Therefore $\lambda$ is a Riesz point of $T$. Since $\lambda \in \mathcal{C} \backslash\{0\}$ was arbitrary, $T \in \mathcal{R}(X)$.

$(2) \Rightarrow(1)$ : We can assume that $K(T) \neq\{0\}$. Since $T \in \mathcal{M}_{0}(X)$ and $0 \in \sigma(T)$ (see [4, Aufgabe 105.2]), 0 is an isolated point of $\sigma(T)$. Hence $K(T)$ is closed (Theorem 4). Put $T_{0}=\left.T\right|_{K(T)}$. By Proposition 1(1), $T(K(T))=K(T)$, thus $T_{0} \in \mathcal{L}(K(T))$. From Proposition 4 we get $K(T)=N\left(P_{0}\right)$. Now use Satz 100.1 in [4] to derive

$$
\sigma\left(T_{0}\right)=\sigma(T) \backslash\{0\},
$$

thus $0 \notin \sigma\left(T_{0}\right)$. Since $T \in \mathcal{R}(X)$ it follows from [4. Satz 105.6] that $T_{0} \in \mathcal{R}(K(T))$. Now assume that $\operatorname{dim} K(T)=\infty$. Thus, by [4, Aufgabe 105.2] $0 \in \sigma\left(T_{0}\right)$, a contradiction. Hence $\operatorname{dim} K(T)<\infty$.

\section{FinAL REMARKS}

1. The proof of Theorem 4 shows that the following result is valid.

Theorem 5. Suppose that $T \in \mathcal{L}(X)$ has the $S V E P$ in 0 and that there is a sequence $\left(\lambda_{n}\right)$ in $\sigma_{p}(T)$ with $\lambda_{n} \neq 0$ for all $n \in \mathbb{N}$ and $\lambda_{n} \rightarrow 0$ as $n \rightarrow \infty$. Then $K(T)$ is not closed.

That the condition " $T$ has the SVEP in 0 " cannot be dropped in Theorem 5 shows the example of the unilateral left shift on $l^{2}(\mathbb{N})$ :

Example. Let $X=l^{2}(\mathbb{N})$, and define the operator $T \in \mathcal{L}(X)$ by

$$
T\left(\xi_{1}, \xi_{2}, \xi_{3}, \ldots\right)=\left(\xi_{2}, \xi_{3}, \ldots\right) .
$$

It is well known that $\sigma_{p}(T)=\{\lambda \in \mathbb{C}:|\lambda|<1\}$. Since $T(X)=X$, we have $K(T)=X$, by [8, Proposition 2]. Thus $K(T)$ is closed. Example 1.7 in [2] shows that $T$ does not have the SVEP in 0.

2. In [1] W. Bouamama proves independently some of the results of our paper.

\section{REFERENCES}

[1] W. Bouamama, Opérateurs de Riesz dont le coeur analytique est fermé, Stud. Math., to appear.

[2] I. Colojoară, C. Foiaş, Theory of generalized spectral operators, Gordon and Breach, New York, 1968. MR 52:15085

[3] J. K. Finch, The single-valued extension property on a Banach space, Pacific J. Math. 58 (1975), 61-69. MR 51:11181

[4] H. Heuser, Funktionalanalysis, 2nd ed., Teubner, Stuttgart, 1986. MR 90m:46001

[5] M. Mbekhta, Généralisation de la décomposition de Kato aux opérateurs paranormaux et spectraux, Glasgow Math. J. 29 (1987), 159-175. MR 88i:47010

[6] M. Mbekhta, Sur la théorie spectrale locale et limite des nilpotents, Proc. Amer. Math. Soc. 110 (1990), 621-631. MR 91b:47004 
[7] W. Gong, L. Wang, Mbekhta's subspaces and a spectral theory of compact operators, Proc. Amer. Math. Soc. 131 (2003), 578-592. MR 2003g:47004

[8] C. Schmoeger, On isolated points of the spectrum of a bounded linear operator, Proc. Amer. Math. Soc. 117 (1993), 715-719. MR 93d:47007

Mathematisches Institut I, Universität Karlsruhe, D-76128 Karlsruhe, Germany

E-mail address: christoph.schmoeger@math.uni-karlsruhe.de 